\title{
Pessary incarcerated in the bladder: A case presentation of vaginal pessary morcellation
}

\author{
Caroline E Foust-Wright ${ }^{1}$, G Sarah Napoe $^{2}$ and Milena M Weinstein ${ }^{3}$ \\ ${ }^{1}$ Clinical Research Fellow, Department of Obstetrics and Gynecology, Massachusetts General Hospital, Boston, MA, USA \\ ${ }^{2}$ Resident, Department of Obstetrics and Gynecology, Massachusetts General Hospital, Boston, MA, USA \\ ${ }^{3}$ Associate Professor, Department of Obstetrics and Gynecology, Massachusetts General Hospital, Boston, MA, USA
}

\begin{abstract}
This is a case of an 82 year-old female with severe pessary neglect who presented 20 years after Gellhorn pessary placement for uterovaginal prolapse with the disc of the pessary found to be incarcerated through a fistulous tract in her bladder. A staged surgical approach included an initial procedure to remove the pessary and place a suprapubic catheter followed by a subsequent vesicovaginal fistula repair. This approach with transection of the pessary to facilitate removal without extending the size of the vesicovaginal fistula and delayed multi-layer closure after 8 weeks of oral estrogen use resulted in full resolution of the fistulous tract.
\end{abstract}

\section{Introduction}

Pelvic organ prolapse affects approximately $1 / 3$ of women in the United States [1]. Treatment options for symptomatic prolapse include pelvic floor physical therapy, pessary use, or surgical intervention. Pessaries are often used in women who either wish to avoid surgical intervention or are poor surgical candidates due to comorbidities. Despite a long history of using mechanical vaginal devices for prolapse reduction, there is no consensus on appropriate management and follow-up after initial pessary fitting [2,3]. Complications from pessary use include vaginal ulceration, bleeding, discharge, need for removal, fistula, discomfort, voiding and defecating dysfunction and incarceration [4]. Complication rates for pessaries are largely unknown, with primarily single-practice settings publishing on local complication rates $[5,6]$. Part of the difficulty in estimating complications from long-term use stem from the lack of management consensus from gynecologic or urogynecologic societies. However, complication rates have been reported as high as $56 \%$ for long-term pessary users (median duration of use 7 years) [6].

\section{Case report}

82 year-old female presented to the emergency department with a one-month history of suprapubic and vaginal pain, exacerbated by standing. In addition, she was having increasing difficulty with urination; initially she had incomplete emptying followed by vaginal leaking with standing which progressed to continuous vaginal leaking. Review of systems was positive for dysuria and hematuria and the ciprofloxacin she was taking for a urinary tract infection was not helping her symptoms. Her medical history was significant for advance pelvic organ prolapse for which a Gellhorn pessary was placed approximately 20 years prior. As she had no difficulties with the pessary, she did not return for follow-up and received no interval pessary care. In the $\mathrm{ED}$, vaginal exam revealed a stricture, above which the stem of the pessary could be palpated along the anterior vaginal wall. A voiding cystourethrogram confirmed a vesicovaginal fistula (VVF) and a CT abdomen/pelvis demonstrated a pessary in the bladder (Figure 1).
The patient was referred to urogynecology where exam revealed a partially agglutinated vagina. The pessary and VVF defect were able to be palpated vaginally but the pessary was unable to be visualized or removed. An office cystoscopy revealed a Gellhorn pessary filling the bladder and a VVF just above the trigone. The patient was scheduled for a staged surgical procedure including pessary removal and suprapubic catheter placement followed by VVF repair.

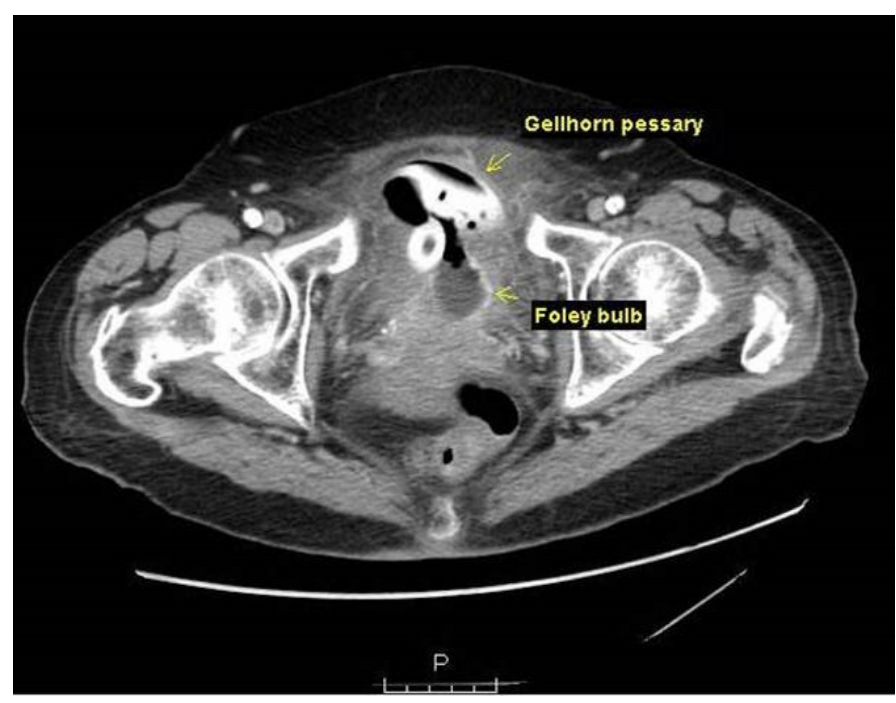

Figure 1. CT scan of pessary in bladder.

Correspondence to: Caroline Foust-Wright, MD, MBA, Department of Obstetrics and Gynecology, Massachusetts General Hospital, 55 Fruit Street, Founders 4, Boston, Massachusetts, USA, Tel: 405-612-1476; Fax: 617-726-4803; E-mail: cfoust-wright@mgh.harvard.edu

Key words: pessary neglect, incarcerated pessary, vesicovaginal fistula

Received: April 04, 2015; Accepted: May 02, 2015; Published: May 05, 2015 
An exam under anesthesia revealed a vaginal stricture $3 \mathrm{~cm}$ above the hymen with the stem of the Gellhorn protruding through the fistula. On cystoscopy, the disc of the Gellhorn was found within the bladder and intravenous Indigo Carmine demonstrated the ureters to be away from the fistulous tract. The cystoscope was left within the bladder to aid with the pessary removal. The vaginal stricture was released with scissors. A tenaculum was then placed on the Gellhorn stem and gentle traction was applied. As the disc of the pessary was too large to be delivered through the VVF tract without extending the fistula, the Gellhorn stem was bisected with scissors and scalpel. While keeping steady traction with the tenaculum, Mayo scissors were used to bisect the disc of the Gellhorn along one side. This allowed the pessary to be folded and removed through the fistula without extending the fistulous tract. Cystoscopy confirmed a $3-4 \mathrm{~cm}$ supratrigonal VVF. A suprapubic tube and Foley catheter were placed for prolonged bladder decompression to reduce surrounding inflammation.

The patient was started on oral estrogen and after five weeks of expectant management, the fistula had reduced in size to approximately 10-12 $\mathrm{mm}$. Eight weeks after her initial procedure, she returned to the operating room for VVF repair. A cystoscopy was performed with the placement of bilateral ureteral stents. Vaginal exam revealed a $15 \mathrm{~mm}$ fistula with eversion of the bladder edges as well as complete agglutination of the upper vagina superior to the fistula. A Latzko vesicovaginal fistula repair with colpocleisis was then performed. First the bladder was hydrodissected off the vaginal edges of the fistula with normal saline. The vaginal edges surrounding the fistula were then dissected off the underlying connective tissue to create flaps. The edges of the fistulous tract were resected and the bladder epithelium was closed with a running suture of 4-0 Monocryl followed by an imbricating layer using 3-0 Monocryl. 2-0 Vicryl was then used to close and imbricate the vagina over the fistula repair. This repair was done in six layers in order to ensure a tension free suture line and reinforce the fistula closure. The suprapubic and urethral Foley catheters were left in place post-operatively for continued bladder decompression.

Post-operatively, the patient was started on tolterodine to prevent bladder spasms and was continued on oral estrogen. She had no vaginal leaking and Methylene Blue injection confirmed no leaking at the fistula site after five weeks with a well-healed vaginal incision. She had no evidence of fistula recurrence, prolapse or bladder dysfunction at her one-year follow-up.

\section{Discussion}

Pessary neglect is likely underreported and complications such as erosion of the pessary into the bladder or rectum can occur as a result. The true incidence of pessary neglect is unknowable, because in order for the neglect to take place, this patient population must necessarily not seek continued medical care unless significant problems arise. A common presentation of pessary neglect is either incidental diagnosis at time of an exam for an unrelated complaint or with a report of vaginal discharge or bleeding. These cases are usually treated with pessary removal and reintegrating the patient into the healthcare field with follow-up arranged with either a gynecologist or urogynecologist. The atypical presentation of urinary or fecal incontinence with subsequent diagnosis of a rectovaginal or vesicovaginal fistula requires more effort to treat and follow-up.

The currently published case reports of pessary removal in these atypical presentations often include perioperative vaginal estrogen as such estrogenized tissue is healthier and therefore easier to manipulate surgically. Removal of the pessary in the operating room under general or spinal anesthesia is a common approach, with a staged procedure planned for repair of the fistulous tract. Gellhorn pessaries are the most commonly used three-dimensional pessaries. Gellhorn pessaries are most commonly reported in cases of fistulas due to pessary neglect. The knob or edges of the ring often are the components of the pessary that erode into the bladder. Due to the shape of the pessary, surgeons frequently enlarge the fistula tract to facilitate the removal of the intact pessary. This increased size of the defect makes the repair more complicated and increases the failure rate of the repair or postoperative complications such as urinary urgency or ureteral compromise.

This case report illustrates the feasibility and utility of bivalving (or hand morcellating) the pessary and removing it in segments to prevent further injury to the bladder. While the Gellhorn pessaries are made of firm silicone or plastic, it is possible to use a combination of cystoscopic and handheld scissors or scalpel to bisect a pessary. This method does not negate the importance of considering a staged procedure to repair the fistula, as allowing improvement in health of vaginal tissue with topical or systemic estrogen prior to repair is vital for successful repair.

\section{References}

1. Hendrix SL, Clark A, Nygaard I, Aragaki A, Barnabei V, et al. (2002) Pelvic organ prolapse in the Women's Health Initiative: gravity and gravidity. Am J Obstet Gynecol 186: 1160-1166. [Crossref]

2. ACOG Committee on Practice Bulletins--Gynecology (2007) ACOG Practice Bulletin No. 85: Pelvic organ prolapse. Obstet Gynecol 110: 717-729. [Crossref]

3. O’Dell K, Atnip S (2012) Pessary care: follow up and management of complications Urol Nurs 32: 126-136, 145. [Crossref]

4. Bugge C, Adams EJ, Gopinath D, Reid F (2013) Pessaries (mechanical devices) for pelvic organ prolapse in women. Cochrane Database Syst Rev 2: CD004010. [Crossref]

5. Arias BE, Ridgeway B, Barber MD (2008) Complications of neglected vaginal pessaries: case presentation and literature review. Int Urogynecol J Pelvic Floor Dysfunct 19: 1173-1178. [Crossref]

6. Sarma S, Ying T, Moore KH (2009) Long-term vaginal ring pessary use: discontinuation rates and adverse events. BJOG 116: 1715-1721. [Crossref]

Copyright: (C2015 Foust-Wright CE. This is an open-access article distributed under the terms of the Creative Commons Attribution License, which permits unrestricted use, distribution, and reproduction in any medium, provided the original author and source are credited. 